EPJ Web of Conferences 27, 00003 (2012)

DOI: $10.1051 /$ epjconf/20122700003

(C) Owned by the authors, published by EDP Sciences, 2012

\title{
Status of GENEUS
}

\author{
V. Wildpaner, D. Neudecker ${ }^{\mathrm{a}}$, Th. Srdinko, and H. Leeb ${ }^{\mathrm{b}}$ \\ Atominstitut, TU Wien, Wiedner Hauptstraße 8-10, 1040 Vienna, Austria
}

\begin{abstract}
The current status of the nuclear data evaluation tool GENEUS (General Nuclear Data Evaluation and Uncertainty System) is discussed. Especially, recent improvements of the implemented algorithms concerning smoothness and stability of the evaluated data. These modifications include an improved extrapolation algorithm for the model defect covariance matrices, the inclusion of uncertainties in level densities and charged particle optical model parameters and the employment of a regularization procedure to restore positive semidefiniteness of the prior covariance matrices. The impact of these improvements is studied for the example of neutron-induced cross sections of ${ }^{55} \mathrm{Mn}$ for which preliminary evaluation results are also provided.
\end{abstract}

\section{Introduction}

The program package GENEUS (General Nuclear Data Evaluation and Uncertainty System) is a nuclear data evaluation tool [1] which provides evaluated cross sections and associated covariance matrices in the energy range between 1 and $200 \mathrm{MeV}$. Hence, the generated data files meet the longstanding demands of the user community regarding an extended energy range (up to $200 \mathrm{MeV}$ ) and the inclusion of uncertainty information. In GENEUS the so-called Full Bayesian Evaluation Technique (FBET) is employed, which is essentially a Bayesian update algorithm adapted to the problem of nuclear data evaluation [2]. In the FBET emphasis is given to a proper determination of the prior, which accounts for the a-priori knowledge of the considered nuclear system. Therefore the FBET is particularly well suited for evaluations which rely strongly on nuclear models because of the scarcity of experimental data.

Following the FBET, nuclear models play an essential role in GENEUS. At present the codes TALYS 1.0 and TALYS 1.2 [3] are implemented and used for the determination of the prior, which comprises cross sections $\sigma_{\eta}(E)$ and covariance matrices $A\left(\eta, E ; \rho, E^{\prime}\right)=\left\langle\Delta \sigma_{\eta}(E) \Delta \sigma_{\rho}\left(E^{\prime}\right)\right\rangle$. Here, $E$ and $E^{\prime}$ refer to the energy of the incident particle and $\eta$ and $\rho$ denote the reaction channel, respectively. According to the FBET the program estimates prior covariance matrices accounting for model defects, $A_{0}^{M D}$, as well as for uncertainties stemming from the limited knowledge of the parameters of the nuclear model, so-called parameter uncertainties $A_{0}^{P U}$. This prior is used in GENEUS as the starting point for the evaluation process employing Bayesian concepts to include consistently available experimental cross section data and associated uncertainty information $[1,2]$. This process leads to evaluated cross sections and covariance matrices which GENEUS also provides in the standard ENDF-6 format.

The first evaluations with GENEUS were presented at the Conference ND2010 [4,5]. For most channels the evaluated cross sections were in fair agreement with the experimental data and the associated error bands were reason-

\footnotetext{
a e-mail: dn@kph.tuwien.ac.at

b e-mail: leeb@kph.tuwien.ac.at
}

able. In addition, we could show for certain reactions that the inclusion of model defects in the prior covariance matrix has a corrective effect on deficient prior cross sections.

Albeit the results are very promising, there are still some features which require either clarification or improvement. One observation concerns the abrupt transition from positive to highly negative correlations in the evaluated covariance matrices, see Fig. 1, which seems to cause problems when including certain experimental data in the evaluation. A second issue concerns reaction channels involving charged particles, where the evaluation frequently exhibits unphysically small error bands and does not follow the included experimental data, but resembles the theoretical cross sections, see e.g. the $(n, p \alpha)$ reaction channel (Fig. 3). In this contribution we will address these points by the example of ${ }^{55} \mathrm{Mn}$.

We consider these problems in Section 2 and present corresponding improvements of the algorithm, i.e. (1) formulate an improved extrapolation algorithm for the model defect covariance matrices, (2) account for uncertainties of level densities and parameters related to charged particles and (3) introduce a regularization procedure to ensure positive semi-definiteness of the covariance matrices. In Section 3 , the improved evaluation of neutron- ${ }^{55} \mathrm{Mn}$ cross sections is shown. At the moment GENEUS is limited to the evaluation of angle-integrated cross section data of structural materials with $A=24$ to $A=209$. In Section 4 further extensions of GENEUS are briefly discussed. A summary and outlook is given in Section 5.

\section{Improved Algorithm}

The unphysical behavior of the evaluated $(n, 2 n)$ correlation matrix, Fig. 1, was attributed in Ref. [1] to the coarse extrapolation algorithm of the model defect covariance matrices $A_{0}^{M D}$. This extension algorithm is needed for energy regions without experimental cross sections of similar isotopes which are essential to estimate $A_{0}^{M D}$ in the formulation of $[2,6]$. In [1], the correlations of the parameter uncertainty covariance matrices $A_{0}^{P U}$ were used to extrapolate $A_{0}^{M D}$. This resulted in discontinuities of $A_{0}^{M D}$ and consequently in pathological correlations of the evaluation, e.g. 


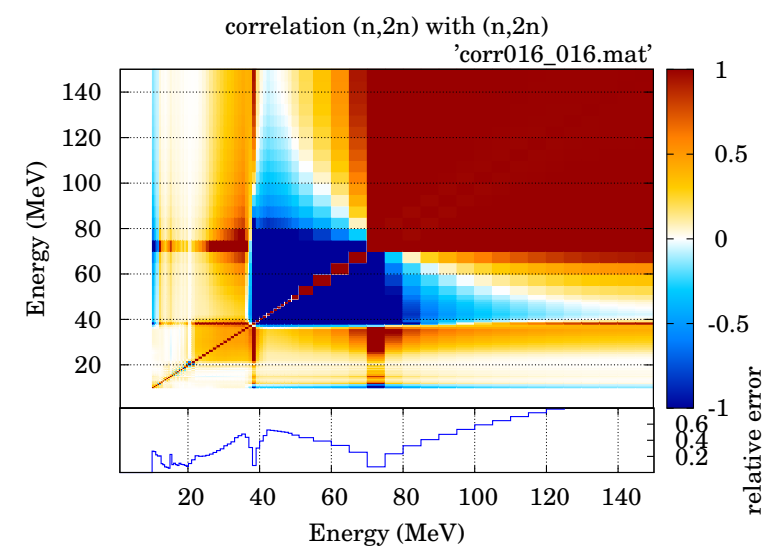

Fig. 1. The correlation matrix and the relative error (graphic below) of the ${ }^{55} \mathrm{Mn}(n, 2 n)$ cross section obtained with GENEUS [1]

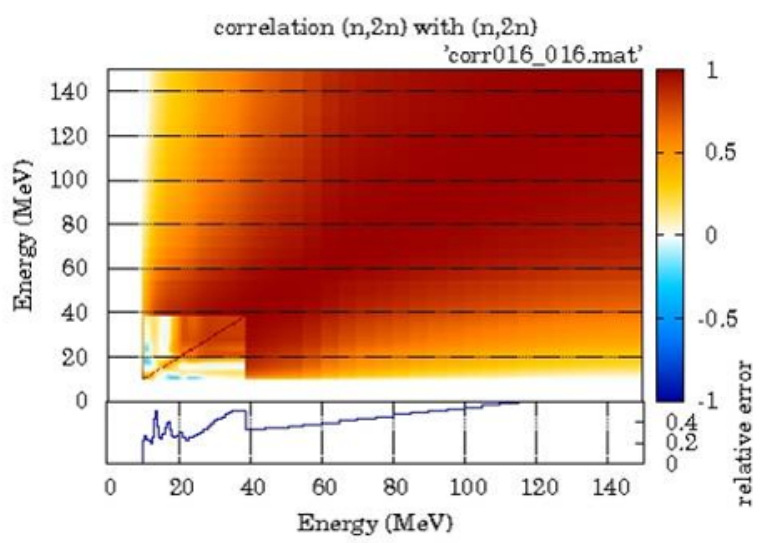

Fig. 2. The correlation matrix and the relative errors (graphic below) of model defects of the ${ }^{55} \mathrm{Mn}(n, 2 n)$ cross section [1].

in the $(n, 2 n)$ reaction channel (Figs. 1 and 2$)$. In order to cure this, a new phenomenological extrapolation algorithm for model defect covariance matrices was developed [6] which guarantees continuous behavior of $A_{0}^{M D}$. Use of the reformulated $A_{0}^{M D}$ results in general in well-behaved evaluated correlation matrices, e.g. for the $(n, 2 n)$ reaction.

Another problem of the evaluation in Ref. [5] occurs in the $(n, p \alpha)$ channel, where the evaluated cross sections are significantly smaller than the experimental ones (Fig. 3). In Ref. [6] this was attributed to the missing model defect covariance matrix for this reaction channel. However, after a detailed study [7] of the parameter space for $A_{0}^{P U}$, this issue could be resolved by expanding the parameter space to include additionally to the neutron optical model parameters also those for the charged particle optical model and level density parameters. Including these additional uncertainties, the evaluated $(n, p \alpha)$ cross sections agree well with the included experimental ones [6].

A major problem of the previous evaluation $[4,6]$ was the fact that not all available experimental data could be included. Including e.g. $(n, p)$ or $(n, \alpha)$ data led to unphysical cross sections, i.e. non-realistic fluctuations and small values below zero. At first glance this phenomenon was attributed to discrepant experimental data. Therefore the experimental data were revisited and their impact studied in detail.

A closer inspection of the $(n, p)$ correlation matrices evaluated with total and elastic data revealed that the un-

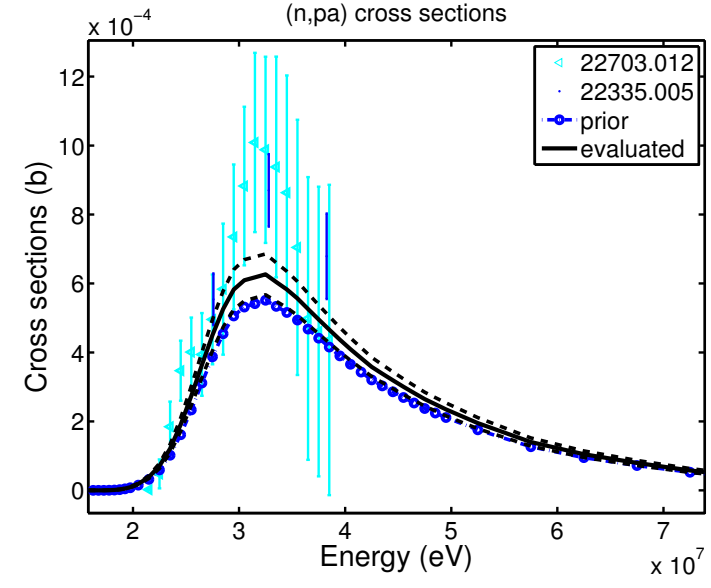

Fig. 3. Comparison of evaluated and prior ${ }^{55} \mathrm{Mn}(n, p \alpha)$ cross section [5] with experimental data.

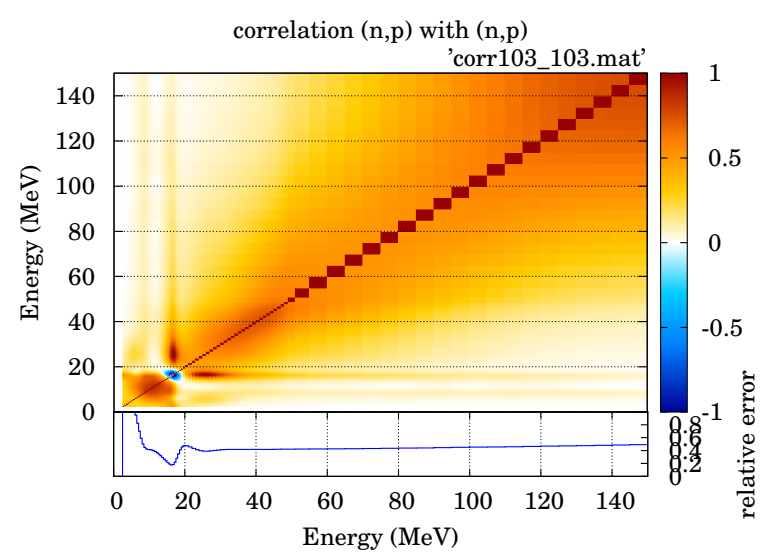

Fig. 4. The evaluated correlation matrix and the relative errors (graphic below) after inclusion of total and elastic data for the ${ }^{55} \mathrm{Mn}(n, p)$ cross section.

physical cross sections were not caused by discrepant experimental data but by the strongly negative correlations of the correlation matrix elements near the diagonal (Fig. 4). If experimental $(n, p)$ data were included with this prior, the evaluated cross sections exhibit a fluctuating behavior reflecting the strongly negatively correlated energy bins in Fig. 4. Neither the well-known and smooth experimental total cross sections nor the elastic ones could be the cause of this strongly negative correlations of neighboring energy bins near the diagonal. Furthermore, none of the evaluated cross sections and covariance matrices of the total and the elastic channel showed any pathological behavior. Thus the prior covariance matrix remained as a potential source of the problem. Therefore we studied the properties of the prior covariance matrix and found a violation of the positive semi-definiteness, a key property of covariance matrices. In general, two cases were observed:

1. For $A_{0}^{P U}$ some very small (smaller than e-13) negative eigenvalues occurred;

2. For $A_{0}^{M D}$ few large negative eigenvalues occurred with absolute values about one-tenth of the size of the largest positive eigenvalue.

The parameter uncertainty covariance matrices $A_{0}^{P U}$ are in general rather stiff, i.e. strongly positively correlated, owing to the fact that they are constructed from model cross 


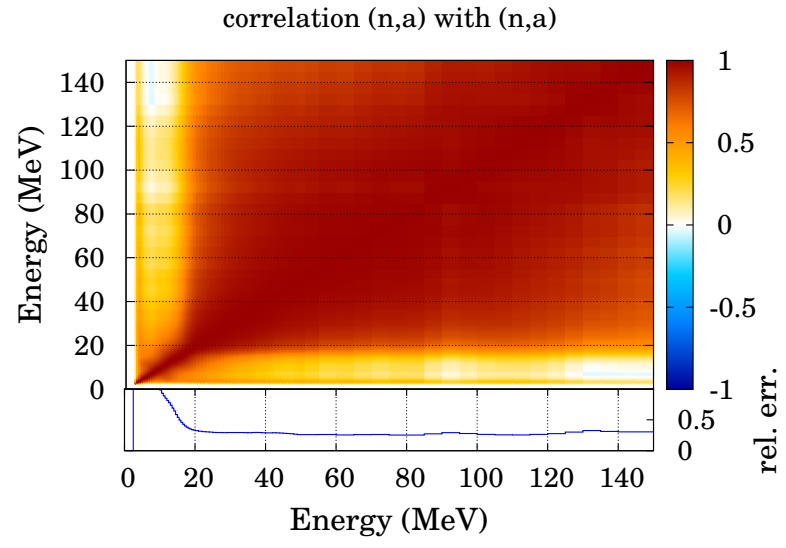

Fig. 5. The correlation matrix and the relative errors (graphic below) stemming from parameter uncertainties of the ${ }^{55} \mathrm{Mn}(n, \alpha)$ cross section before regularization.

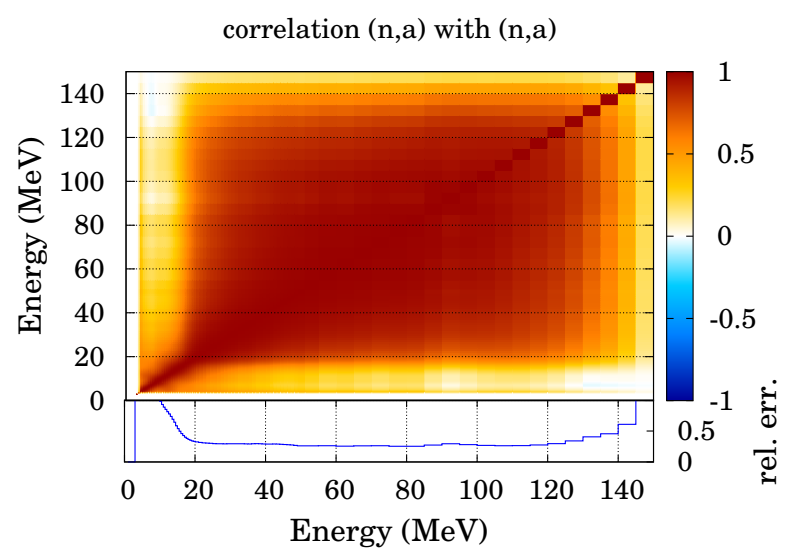

Fig. 6. The correlation matrix and the relative errors (graphic below) stemming from parameter uncertainties of the ${ }^{55} \mathrm{Mn}(n, \alpha)$ cross section after regularization.

sections depending on a set of parameters. These strong positive correlations translate in a larger quantity of eigenvalues which are zero or very small. Numerical instabilities in inversion algorithms as well as rounding errors could result in several very small negative eigenvalues and are thus a numerical artifact. These small negative eigenvalues are eliminated by adding a small positive number of the size of the negative eigenvalues to all diagonal elements of $A_{0}^{P U}$. This weakens slightly the correlations between different energy bins and restores the required positive semidefiniteness. In case of ${ }^{55} \mathrm{Mn}$, the only visible impact of the regularization of $A_{0}^{P U}$ occurs in the $(n, \alpha)$ reaction channel (see Figs. 5 and 6), where the correlations above $120 \mathrm{MeV}$ are weakened. However, in this energy range the cross sections are almost zero.

The situation is different for $A_{0}^{M D}$ because there occur few large negative eigenvalues which cannot be caused by numerical instabilities but indicate unphysical behavior. The problem is evident in the $(n, 3 n)$ channel (Fig. 7). Starting from about $30 \mathrm{MeV}$, neighboring energy bins near the diagonal are negatively correlated over a large energy range, which is unphysical. This is caused by experimental $(n, 3 n)$ data used to estimate $A_{0}^{M D}$. In order to extract the unphysical information, we calculate the eigenvalue repre-

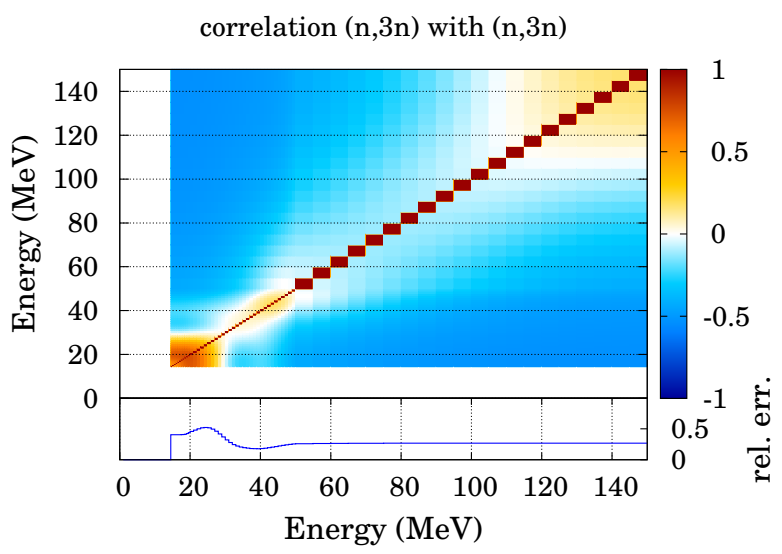

Fig. 7. The correlation matrix and the relative errors (graphic below) stemming from model defects of the ${ }^{55} \mathrm{Mn}(n, 3 n)$ cross section before regularization.

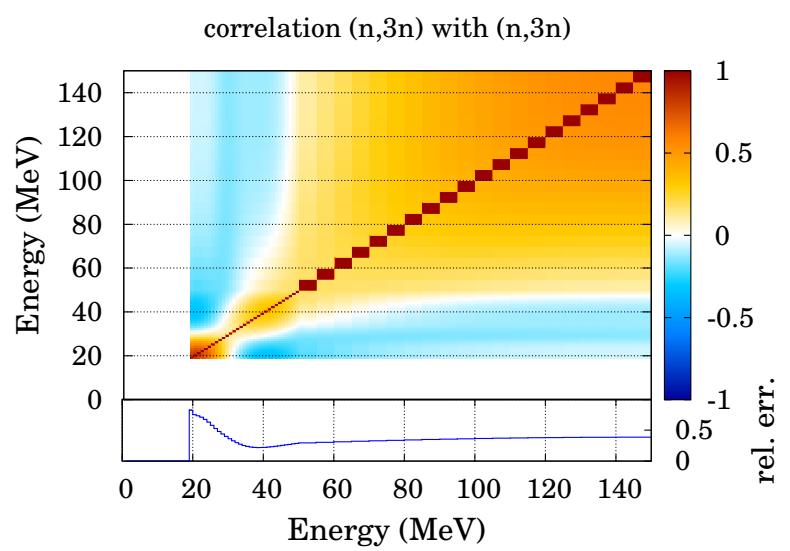

Fig. 8. The correlation matrix and the relative errors (graphic below) stemming from model defects of the ${ }^{55} \mathrm{Mn}(n, 3 n)$ cross section after regularization.

sentation $D_{0}^{M D}$ of $A_{0}^{M D}$

$$
D_{0}^{M D}=Q^{T} A_{0}^{M D} Q,
$$

and generate a regularized diagonal matrix $\tilde{D}_{0}^{M D}$ which has the same eigenvalues as $D_{0}^{M D}$, except the negative ones which are substituted with zero. The regularized model defect covariance matrix is then given by

$$
\tilde{A}_{0}^{M D}=Q \tilde{D}_{0}^{M D} Q^{T} .
$$

Applying this procedure to $A_{0}^{M D}$ of $(n, 3 n)$, Fig. 7, results in a more reasonable covariance matrix, Fig. 8.

Consequently, the regularized prior covariance matrix $\tilde{A}_{0}$, which is a nonnegative linear combintation of $\tilde{A}_{0}^{P U}$ and $\tilde{A}_{0}^{M D}$, is also positive semi-definite, see Ref. [8]. The experimental covariance matrices were also checked for positive semi-definiteness. In general, it was only violated in case of erroneous input data. Using regularized and improved prior covariance matrices in the evaluation led to reasonable evaluation output, which will be shown in Section 3 for neutron ${ }^{55} \mathrm{Mn}$ reactions.

Another issue which should be addressed is to get a formally correct ENDF-6 output file. In order to guarantee this we implemented the ENDF-6 Checking and Utility Codes (FIZCON, CHECKR, INTER and PYSCHE) of [9]. 
Table 1. Level density parameters for the Constant Temperature model implemented in TALYS 1.0 [3] and associated boundares for $A_{0}^{P U}$.

\begin{tabular}{llllll}
\hline$\alpha$ & $\alpha^{>}[\%]$ & $\alpha^{<}[\%]$ & $\beta$ & $\beta^{>}[\%]$ & $\beta^{<}[\%]$ \\
\hline 0.02622 & 45.0 & 45.0 & 0.27042 & 45.0 & 45.0 \\
\hline$\gamma_{1}$ & $\gamma_{1}^{>}[\%]$ & $\gamma_{1}^{<}[\%]$ & $\delta_{\text {global }}$ & $\delta_{\text {global }}^{>}$ & $\delta_{\text {global }}^{<}$ \\
\hline 0.4563 & 30.0 & 30.0 & 0.0 & 3.0 & -3.0 \\
\hline
\end{tabular}

\section{Improved Evaluation of ${ }^{55} \mathrm{Mn}$}

\subsection{A-priori Information}

The a-priori information of the Full Bayesian Evaluation Technique is supplied by GENEUS in form of cross sections and covariance matrices. For the prior cross sections, the same nuclear model with the same parameterization as in [5] was employed. For the estimation of $A_{0}^{P U}$, the parameter boundaries defined in [7] were used for the neutron and charged particle optical potential parameters. The boundaries for the level density parameters are given in Table 1. Their admissible ranges are larger than in [7] and were established such that the prior uncertainties include experimental data. The model defect covariance matrices $A_{0}^{M D}$ were estimated and extrapolated according to [6]. The matrix $A_{0}^{P U}$ as well as $A_{0}^{M D}$ were regularized following the procedures in Section 2 and combined to a prior covariance matrix according to [6]. Model cross sections were calculated using TALYS 1.0 [3] and the experimental knowledge was extracted from EXFOR [10].

\subsection{Evaluation and Discussion of Results}

A total number of 792 experimental cross sections ranging from 1 to $150 \mathrm{MeV}$ of nine different channels were included in three update steps into the prior knowledge via the FBET [1,2]. The literature and information provided in EXFOR [10] for each experimental data set of Table 2 was carefully studied to estimate associated experimental covariance matrices. Correlations between different experiments were also taken into account in a coarse manner. It is implicitly assumed that experimental data of different update steps are uncorrelated. This might be true for the data of the first update step. However, the data of the second and third update step might have non-zero correlations. They were treated in different update steps as for the reaction channels of the third update no model defect covariance matrices could be estimated, whereas they were available for the reaction channels of the second update step. The evaluated cross sections of several channels are shown in Figs. 9 to 15. All evaluated cross sections and associated error bars correspond well to the included experimental information. It should be noted that it was now possible to include experimental data of all channels without unphysical artifacts. This progress has only been achieved by the regularization of the prior outlined in Section 2. Unfortunately, there are no experimental $(n, 3 n)$ cross sections available.

The small kink of the evaluate total cross sections, Fig. 9, is caused by the structure of the experimental data. The kinks in the evaluated $(n, \alpha)$ cross sections reflect the slightly
Table 2. Table 2. List of experimental data (cited according to their EXFOR [10] number) included in the evaluation of ${ }^{55} \mathrm{Mn}$. The channel, total number of data points used in the evaluation and the corresponding update step are also given.

\begin{tabular}{llcrc}
\hline First author & EXFOR & channel & \# data & update \\
\hline Abfalterer & 13753.018 & total & 333 & 1 \\
Foster & 10047.031 & total & 248 & 1 \\
Nereson & 11308.008 & total & 30 & 1 \\
Manero & 20169.002 & total & 62 & 1 \\
Schweitzer & 30463.042 & elastic & 1 & 1 \\
Holmqvist & 20019.081 & elastic & 8 & 1 \\
\hline Glazkov & 40680.019 & inelastic & 2 & 2 \\
Fujita & 21722.027 & inelastic & 1 & 2 \\
Bostan & 22292.008 & $(n, p)$ & 7 & 2 \\
Bostan & 22292.006 & $(n, 2 n)$ & 3 & 2 \\
Menlove & 11421.005 & $(n, 2 n)$ & 10 & 2 \\
Filatenkov & 41240.010 & $(n, 2 n)$ & 7 & 2 \\
Filatenkov & 41298.011 & $(n, 2 n)$ & 1 & 2 \\
Uwamino & 22703.011 & $(n, 2 n)$ & 28 & 2 \\
Bostan & 22292.007 & $(n, \alpha)$ & 7 & 2 \\
Filatenkov & 41298.010 & $(n, \alpha)$ & 9 & 2 \\
Fessler & 22414.016 & $(n, \alpha)$ & 5 & 2 \\
\hline Sudar & 30473.003 & $(n, t)$ & 2 & 3 \\
Soewarsono & 22335.007 & $(n, 4 n)$ & 1 & 3 \\
Uwamino & 22703.010 & $(n, 4 n)$ & 6 & 3 \\
Soewarsono & 22335.005 & $(n, p \alpha)$ & 3 & 3 \\
Uwamino & 22703.012 & $(n, p \alpha)$ & 18 & 3 \\
\hline
\end{tabular}

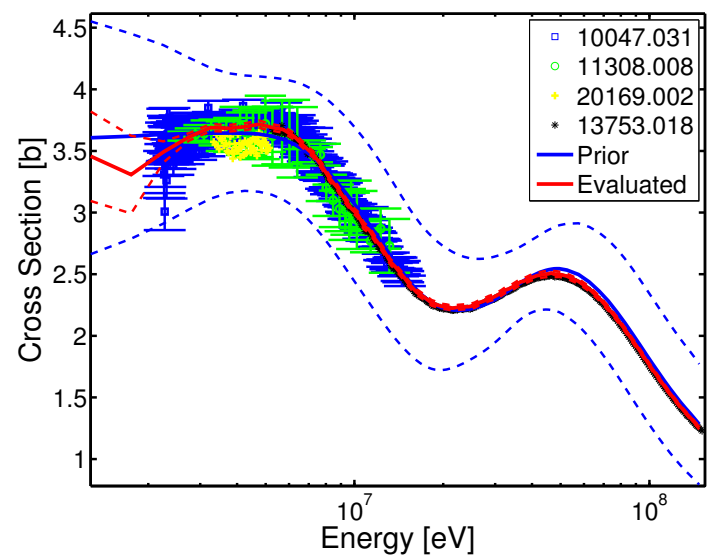

Fig. 9. Comparison of evaluated, experimental and prior total neutron- ${ }^{55} \mathrm{Mn}$ cross sections.

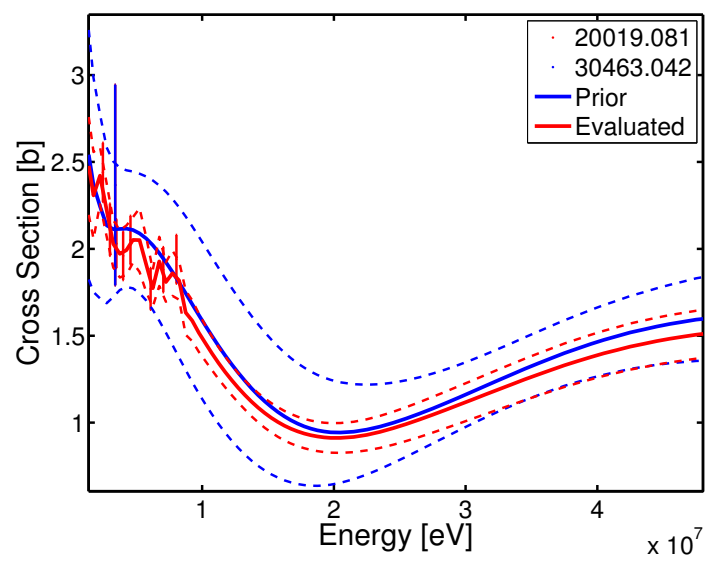

Fig. 10. Comparison of evaluated, experimental and prior elastic neutron- ${ }^{55} \mathrm{Mn}$ cross sections. 


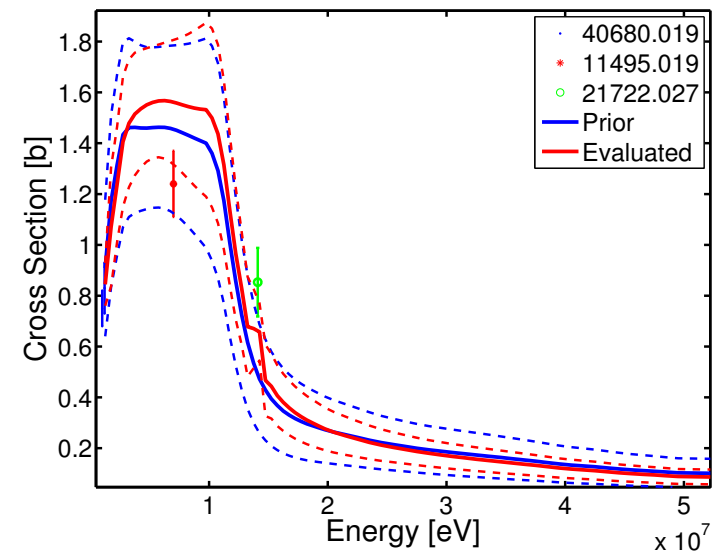

Fig. 11. Comparison of evaluated, experimental and prior inelastic neutron- ${ }^{55} \mathrm{Mn}$ cross sections.

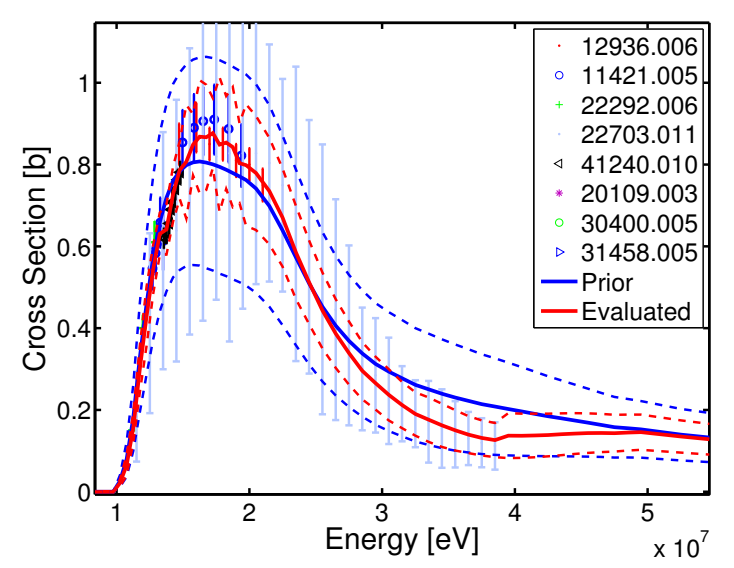

Fig. 12. Comparison of evaluated, experimental and prior ${ }^{55} \mathrm{Mn}(n, 2 n)$ cross sections.

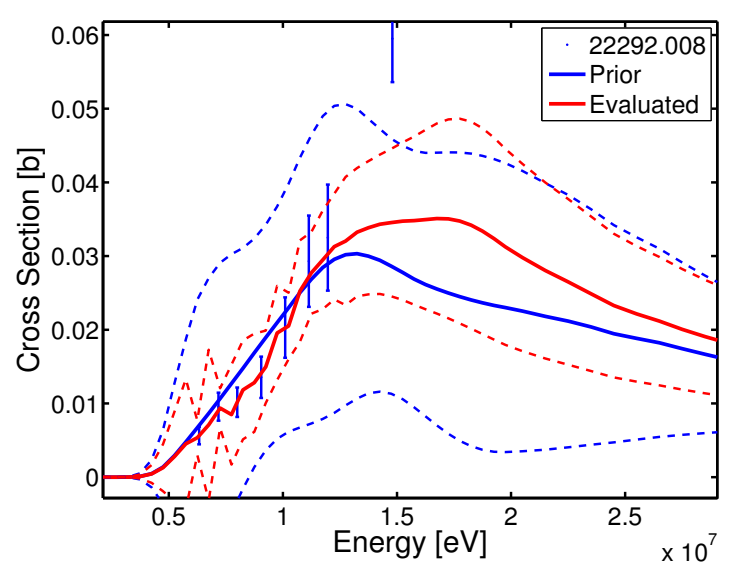

Fig. 13. Comparison of evaluated, experimental and prior ${ }^{55} \mathrm{Mn}(n, p)$ cross sections.

discrepant experimental data and their pronounced difference from the prior cross sections (Fig. 14). These unphysical kinks are caused by weak prior correlations. In principle, $\tilde{A}_{0}^{P U}$ in Fig. 6 is stiff but the dominant $\tilde{A}_{0}^{M D}$ is weakly correlated corresponding to the deficient prior cross sections. At the moment studies are undertaken to conserve

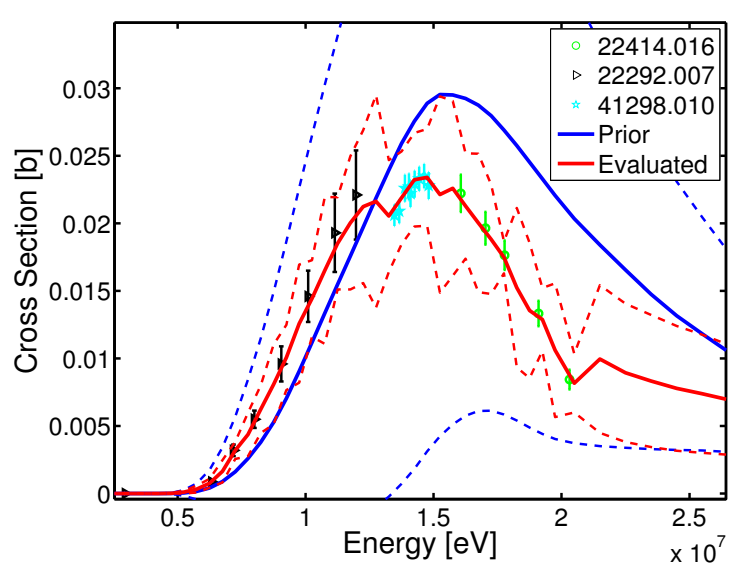

Fig. 14. Comparison of evaluated, experimental and prior ${ }^{55} \mathrm{Mn}(n, \alpha)$ cross sections.

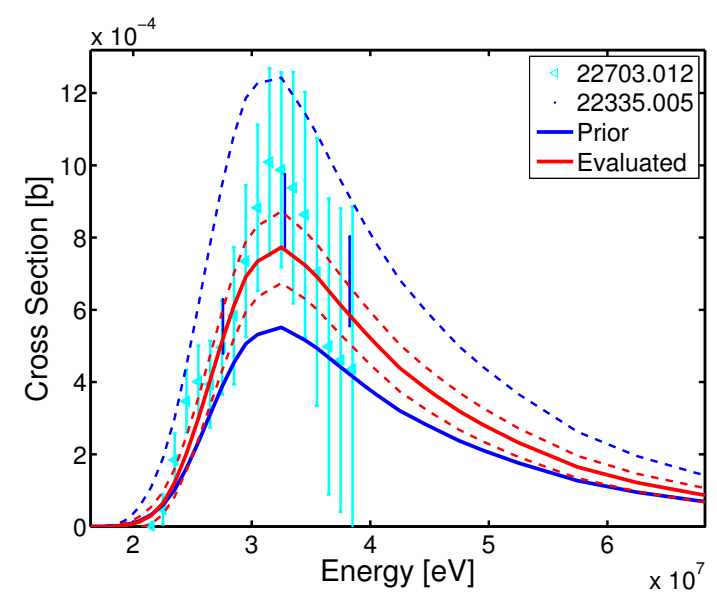

Fig. 15. Comparison of evaluated, experimental and prior ${ }^{55} \mathrm{Mn}(n, p \alpha)$ cross sections.

the smoothness of the evaluated cross sections corresponding to physics expectations by means of prior information.

Of special interest are the $(n, 2 n),(n, \alpha)$ and $(n, p \alpha)$ reaction channels as the prior cross sections did not describe the experimental data well. However, the new evaluated data agree with the experiments within the error bars. In the case of the $(n, 2 n)$ reaction channel this behavior is even sustained in energy ranges without experimental information. This behavior of $(n, 2 n)$ is only possible if $A_{0}^{M D}$ is included in the evaluation process, see e.g. [1,4]. The evaluated $(n, p \alpha)$ cross sections are also of great interest as they reproduce experimental data much better than those of Fig. 3 which were much too low. This correction of the evaluated output was enabled by the enlargement of the parameter space for $A_{0}^{P U}$ to enclose uncertainties of all important parameters for $(n, p \alpha)$.

No figures for the $(n, t),(n, 3 n)$ and $(n, 4 n)$ cross sections are given here. They correspond well to the few data points available for the $(n, 4 n)$ and $(n, t)$ reaction channels and are smooth functions with behavior similar to the theoretical cross sections.

The evaluated covariance matrix as well as each covariance matrix after one update step are positive semidefinite. Here, only the $(n, 2 n ; n, 2 n)$ evaluated correlation matrix is given in Fig. 16 in order to show the improvement compared to the one of Ref. [1] (Fig. 1). It is of a 
reasonable structure and does not show any longer discontinuities as in Fig. 1. This improvement is a consequence of the new extrapolation algorithm for $A_{0}^{M D}$ as well as by restoring positive semi-definiteness of the prior covariance matrices. Hence, we achieved a significant improvement of the algorithm of the FBET and provide reasonable evaluated output for all considered channels. Figures of the other covariance matrices can be obtained on request from the authors. They are also continuous and have a reasonable structure.

\section{Future Developments}

The present version of GENEUS is limited to structural materials with mass numbers $A$ between 24 and 209. Currently we work on an extension to fissile materials. Therefore we have implemented TALYS 1.2 [3] into GENEUS, in order to calculate also fission observables. At present we study the model parameters associated with fission and the proper parameter space required for $A_{0}^{P U}$. Preliminary studies for ${ }^{241} \mathrm{Am}$ and ${ }^{239} \mathrm{Pu}$ gave first indication that the fission barrier and width have a significant impact on the fission cross sections, while the other fission parameters of TALYS [3] seem to have a minor impact. Currently, further studies are in progress in order to verify this properties and to define reasonable parameter boundaries.

In addition we have included the MT-numbers relevant for fission in $M F=3$, i.e. $M T=18-21$ and 38, as well as $\mathrm{MF}=12$ and $\mathrm{MF}=4$. The inclusion of further MF-files is currently under development.

The treatment of angle-differential data in the evaluation process of GENEUS is envisaged in the near future.

\section{Summary and Outlook}

At the conference ND2010 the first version of GENEUS was presented [1]. Albeit the first applications to ${ }^{55} \mathrm{Mn}$ and ${ }^{208} \mathrm{~Pb}$ already indicated its applicability, some open issues remained. One issue concerned pathological behavior of evaluated correlation matrices with discontinuous transitions between positive and strongly negative correlations (Fig. 1). Another issue were the much too low evaluated ( $n, p \alpha)$ cross sections, see Fig. 3. Furthermore, including some experimental cross sections in the evaluation resulted in pathological evaluated output. We investigated the occurrence of these phenomena and formulated modified algorithms to solve these problems. Especially we developed a new extrapolation algorithm and reformulation of the model defect covariance matrices (see also Ref. [6]), expanded the parameter space of the parameter uncertainty covariance matrix (see also Ref. [4]) and introduced a regularization procedures to restore positive semi-definiteness of these covariance matrices (see Section 2).

These improvements were applied to the prior knowledge of neutron- ${ }^{55} \mathrm{Mn}$ reactions. Employing this improved prior knowledge results in continuous and reasonable evaluated covariance matrices, Fig. 16. The evaluated cross sections agree also with the included experimental data, Figs. 9 to 15. Furthermore, experimental data for all reaction channels could now be included without resulting in unphysical output. Hence, we significantly improved the

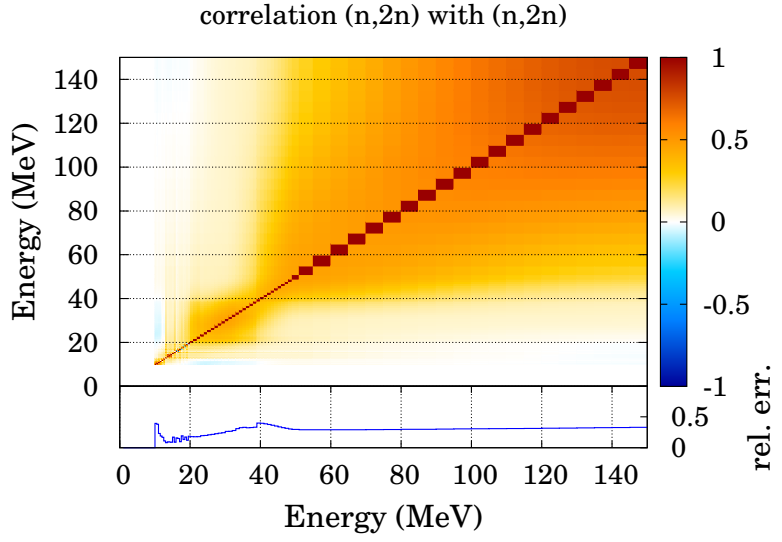

Fig. 16. The evaluated correlation matrix and associated relative errors (graph below) of the ${ }^{55} \mathrm{Mn}(n, 2 n)$ cross sections.

algorithms in GENEUS which allows us to generate reasonable evaluations for all considered reaction channels.

At the moment, developments of GENEUS are undergoing to include fission reaction channels and associated ENDF-6 output files. The first studies and developments have been briefly summarized. Furthermore, studies are undertaken to conserve the smoothness of the cross sections in the evaluation following physics expectations.

This work was partly supported by the EURATOM project ANDES. The views and opinions expressed herein do not reflect necessarily those of the European Commission. The computational results presented have been achieved in part using the Vienna Scientific Cluster (VSC).

\section{References}

1. H. Leeb, St. Gundacker, D. Neudecker, Th. Srdinko, V. Wildpaner, J. Korean Phys. Soc. 59 No. 2, (2011) 959

2. H. Leeb, D. Neudecker, Th. Srdinko, Nucl. Data Sheets 109, (2008) 2762

3. A.J. Koning, S. Hilaire, M.C. Duijvestijn, Proceedings of the International Conference on Nuclear Data for Science and Technology ( EDP Sciences, Nice 2008) 211

4. D. Neudecker, St. Gundacker, Th. Srdinko, V. Wildpaner, H. Leeb, J. Korean Phys. Soc. 59 No. 2, (2011) 1272

5. H. Leeb, St. Gundacker, D. Neudecker, Th. Srdinko, V. Wildpaner, J. Korean Phys. Soc. 59 No. 2, (2011) 1230 .

6. H. Leeb, St. Gundacker, D. Neudecker, Th. Srdinko, V. Wildpaner, Proceedings of Final Scientific EFNUDAT Workshop (Cern, Geneva 2010) 55

7. D. Neudecker, St. Gundacker, Th. Srdinko, V. Wildpaner, H. Leeb, Proceedings of Final Scientific EFNUDAT Workshop (Cern, Geneva 2010) 49

8. R.A. Horn, C.R. Johnson, Matrix Analysis (Cambridge Univ. Press, Cambridge 1985)

9. M.B, Chadwick et al., Nucl. Data Sheets 107, (2006) 2931

10. IAEA Nuclear Data Section, EXFOR Formats Description for Users (IAEA-NDS-206, Vienna 2008) 